\title{
Feasibility study of a shielding-independent radiation portal monitor system for revealing 241-Am orphan sources in radiometric surveillance of scrap metal
}

\author{
Gabriele Zorloni $^{\mathrm{a}}{ }^{\mathbb{D}}$, Paolo Tancioni, Marco Caresana \\ Department of Energy, Politecnico di Milano, via Lambruschini 4, 20156 Milan, Italy
}

Received: 10 July 2020 / Accepted: 2 September 2020 / Published online: 7 September 2020

(C) The Author(s) 2020

\begin{abstract}
Orphan radioactive sources inadvertently melted in steel plants are a health and economical hazard. Thus, regulation authorities impose strict control measurements to be performed at these plants. However, a recent series of incidents related to the melting of ${ }^{241} \mathrm{Am}$ sources demonstrated the inadequacy of even state of the art systems in revealing this low-energy gamma ray emitter up to activities of the order of hundreds GBq. However, ${ }^{241}$ Am sources are characterized by a weak neutron emission which can be detected by proper monitor systems. This work provides a feasibility study of a shielding-independent neutron-sensitive portal monitor system to be installed in steel plants. Measurements were performed both with a certified ${ }^{241} \mathrm{Am}$ shielded source and at the weighing area of a steel plant, to assess both the detector performance and the practical feasibility of the system implementation in work-place fields. A risk evaluation analysis demonstrated that with the current control systems ${ }^{241} \mathrm{Am}$ sources up to $185 \mathrm{GBq}$ could pass undetected with a potential exposure for workers equal to $1.7 \mathrm{mSv}$, consistent with recent accidents reports. The proposed technique could reveal ${ }^{241} \mathrm{Am}$ activities down to $2 \mathrm{GBq}$, decreasing the worker exposure down to $24 \mu \mathrm{Sv}$, without interfering with the standard plant operations.
\end{abstract}

\section{Introduction}

The melting of radioactive orphan sources in furnaces of steel plants is one of the major causes of incidents related to radioactive materials not connected with trafficking or malicious use [1]. For this reason, national and international regulatory authorities impose different radiometric surveys for the scrap metal industry. The most common radioactive sources involved in steel plants incidents are ${ }^{60} \mathrm{Co},{ }^{137} \mathrm{Ce}$ and ${ }^{226} \mathrm{Ra}$. Less frequent cases involve the melting of ${ }^{241} \mathrm{Am}$ sources. However, the Outokumpu case is a significant representative series of recent events.

Outokumpu is a Finnish firm which operates in the steel production and metallurgical industry. In 2012, a ${ }^{241}$ Am source was melted in the Finnish plant of Tornio. The radiological alarm was given by detectors screening the slag, and four workers are considered to have been exposed to ${ }^{241} \mathrm{Am}$ through inhalation. One of the employees is thought to be exposed to $10 \mathrm{mSv}$ [2]. The event was qualified as INES (International Nuclear and radiological Event

a e-mail: gabriele.zorloni@ polimi.it (corresponding author) 
Scale) Level 1. After that, other four accidents occurred in 2018 within a period of 4 months involving the melting of scrap containing americium. All these cases concerned different European Outokumpu facilities [3]. The activities involved in these incidents were of the order of the $\mathrm{Ci}$ (tens-hundreds $\mathrm{GBq}$ ), and the recurrence of these events qualified them as INES Level 1 incidents.

As far as ${ }^{241} \mathrm{Am}$ is concerned, firms involved in the metal scrap industry all agree on the difficulties implied by detecting these sources because of their low energy gamma emission (59.5 keV). Even high-quality Radiation Portal Monitor (RPM) systems mounting large slabs of plastic scintillators can hardly detect even high activity ${ }^{241}$ Am sources. Nowadays, the risk of inadvertently melting americium is somehow accepted, and it is impossible to calculate the number of these sources that have been already melted and dispersed in the environment without triggering any alarm.

${ }^{241} \mathrm{Am}$ sealed sources are generally made of Am oxides compounds . Typical activities range from tens $\mathrm{MBq}$ up to hundreds GBq, and generally have a working life of 15-20 years [4]. A peculiar aspect of all radioactive sources containing americium oxides is their high energy neutrons emission due to $(\alpha, \mathrm{n})$ reactions on oxygen. ${ }^{241} \mathrm{Am}$ sources manufacturers declare a neutron emission up to about $10^{3}$ neutrons per second per GBq activity of the source [4].

Even though the relatively weak neutron emission of ${ }^{241}$ Am sealed sources, a RPM system equipped with proper neutron detectors might be able to reveal even low activity sources. Differently from the low-energy gamma rays, high-energy neutrons are not stopped in the surrounding metal and can reach the detector without significant attenuation.

In this work, we present a feasibility study of a RPM system capable of revealing ${ }^{241} \mathrm{Am}$ activities down to $2 \mathrm{GBq}$ over the $90 \%$ of the load volume to be installed at the entrance of a steel plant. A ${ }^{3} \mathrm{He}$-free gas-scintillator neutron detector was used as a representative instrument to be employed for this application. Measurements were performed both with a lead shielded certified ${ }^{241} \mathrm{Am}$ source and at the weighing area of a steel plant, to evaluate both the critical level of detectable activity and the feasibility of such a system to be employed in real-case scenarios, both in terms of spatial footprint and procedural time requirements. A preliminary risk evaluation was also performed to assess the impact of such system to the safety of workers.

\section{Materials and methods}

\subsection{Neutrons from americium sources}

${ }^{241} \mathrm{Am}$ sealed sources are usually made of Am oxides compounds, in general in the form of $\mathrm{AmO}_{2}$, encapsulated in ceramic enamel and sealed in stainless steel capsules [5]. $(\alpha, \mathrm{n})$ reactions took place on oxygen and on the surrounding low atomic number elements such as $\mathrm{Al}, \mathrm{Si}, \mathrm{Mg}$, and $\mathrm{F}$ [6]. The most probable $(\alpha, \mathrm{n})$ reaction for ${ }^{241} \mathrm{Am}$ oxide sources involves ${ }^{18} \mathrm{O}$, emitting a broad energy neutron spectrum with a maximum energy equal to about 4.2 $\mathrm{MeV}$.

For neutron energies of the order of the $\mathrm{MeV}$, the total cross section of iron is of the order of 3-5 b [7]. The absorption cross section, i.e. $(\mathrm{n}, \gamma)$ and $(\mathrm{n}, \mathrm{e})$, is of the order of the $\mathrm{mb}$, while the remaining is almost due to elastic scattering processes. Elastic scattering, which does not directly lead to the neutron loss, mainly causes i) energy degradation of the primary field, and ii) build-up. This means that, differently from the low energy ${ }^{241} \mathrm{Am}$ gamma rays, ferrous 
scrap material can be considered quasi-transparent to the neutrons emitted by the source, or at least, it does not easily absorb the primary field.

The absorption cross section of lead, which in principle could be found in the scrap in the case of shielded sources, in the same energy range is again a few millibarns [7]. It is worthwhile noting that even for relatively intense ${ }^{241} \mathrm{Am}$ sources, few millimeters of lead completely stop the $59.5 \mathrm{keV}$ gamma emission, while several centimeters of the material are again transparent to neutrons. A neutron detector based RPM system could, in principle, reveal both shielded and unshielded ${ }^{241}$ Am sources, while standard RPM system is almost completely blind in the case of a shielded source.

\subsection{Neutron detector}

The neutron detector used is an ARKTIS M800 neutron detector (ARKTIS Radiation Detectors, Switzerland). It consists of a ${ }^{4} \mathrm{He}$ filled tube placed inside a high density polyethylene (HDPE) hollow parallelepiped which acts as a moderator. The device is based on the gaseous scintillation principle. The moderator is optimized for safeguards applications, in particular for a bare ${ }^{252} \mathrm{Cf}$ point source.

A proprietary large area ${ }^{6} \mathrm{Li}$-impregnated polymer is placed inside the detector tube acting as the neutron sensitive material. After thermal neutron capture, lithium emits high-energy charged particles, thus causing the gas scintillation. Light is detected by a series of silicon photomultipliers (SiPMs). The detector (about $1 \mathrm{~m}$ long and $20 \mathrm{~cm}$ in diameter) is subdivided in eight independent sectors, each of them read by 3 independent SiPMs. Analog-to-digital conversion is performed on-board. The output information consists of a $8 \times 192$ matrix transferred each 10 seconds. Each row corresponds to a sector. Each column corresponds to an arbitrary 192 bin histogram in time-over-threshold (ToT) discretized amplitudes units. Thus each independent sector performs pulse amplitude spectrometry. The basic matrix information is sent via Ethernet to a laptop together with other detector information. Measurement duration and parameters setting (i.e. SiPMs overvoltage, ToT low threshold etc. ${ }^{1}$ ) are software controlled. The detector is powered via Power over Ethernet.

For each $10 \mathrm{~s}$, the output $\log$ file is updated. Each update consists of a series of detector diagnostic information plus the counts matrix. The log file permits to reconstruct the whole measurement history with a 10-s time discretization. The key parameter to be optimized during the analysis is the signal-to-background ratio (S/B), meaning to find the best Region Of Interest (ROI) which optimizes the device sensitivity to the source with respect to the background counts. The high energy neutrons and the gamma rays due to natural and cosmic radiation cause a slightly different histogram output with respect to a quasi-pure neutron field, thus a ROI optimization procedure can be performed.

This type of detector was chosen considering the high efficiency, the presence of a moderating box designed for ${ }^{252} \mathrm{Cf}$ detection (the mean neutron energy of ${ }^{252} \mathrm{Cf}$ is similar to the ${ }^{241} \mathrm{Am}$ oxide case here studied), low power requirements and robustness. The producer declares a sensitivity of about $1.05 \mathrm{cps} / \mathrm{ng}{ }^{252} \mathrm{Cf}$ at $2 \mathrm{~m}\left(1 \mathrm{ng}{ }^{252} \mathrm{Cf}\right.$ emits roughly 2300 neutrons per second). Robustness is achieved considering the absence of crystals, photomultiplier tubes and high-voltage power supplies, and the inert nature of the inner gas. A high-efficiency and robust detector is mandatory for a steel plant scenario, so M800 can be

\footnotetext{
${ }^{1}$ Even though these parameters play a role in the evaluation of the detector performance, in this study they were kept equal to the values suggested by the producer. This choice was made to ensure that the detector was correctly working in its standard working conditions, since the fine-tuning of these parameters is beyond the scope of this feasibility study.
} 
Fig. 1 Picture of the experimental setup at the calibration facility of Politecnico di Milano. The neutron detector and the control laptop are shown on the right. The irradiator is shown on the left at $4 \mathrm{~m}$ from the detector

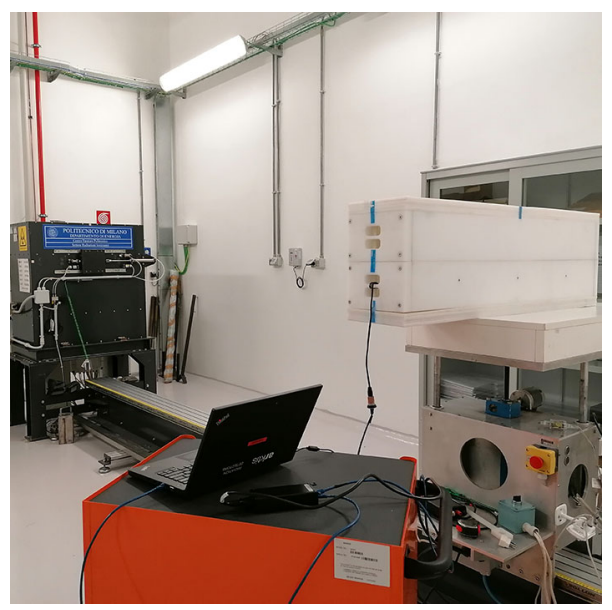

considered as a representative of a potential class of neutron detectors to be employed for this application.

\subsection{Measurements description}

Measurements were performed in two different sites. Firstly, the detector sensitivity to a shielded ${ }^{241}$ Am source was investigated at a calibration facility in controlled conditions. Secondly, a series of measurements were performed directly at the entrance of a steel plant without the presence of the radioactive source.

\subsubsection{Measurements in laboratory conditions}

For testing the detector efficiency to a shielded ${ }^{241}$ Am source, an experiment was designed in the calibration facility of Politecnico di Milano, Italy. The facility hosts a self-shielded irradiator containing a certified isotopic $37 \mathrm{GBq}^{241} \mathrm{Am}$ source. The irradiator is provided with a moving platform which permits to place the detector from $1 \mathrm{~m}$ up to $4 \mathrm{~m}$ from the source. A laser pointing system permits the positioning of the detector in the desired position with an uncertainty of $\pm 1 \mathrm{~mm}$. A 5-cm lead shutter at the irradiator exit window completely stops the gamma rays of the ${ }^{241} \mathrm{Am}$ source, but it is not designed to stop neutrons. This condition can be considered representative of a $37 \mathrm{GBq}^{241} \mathrm{Am}$ source strongly shielded within a full-load truck. A picture of the experiment is shown in Fig. 1.

Measurements were performed at $1 \mathrm{~m}, 2 \mathrm{~m}$, and $4 \mathrm{~m}$ from the shielded source. Acquisition time was set equal to ten minutes, which was observed to be adequate for obtaining good statistics for the data analysis.

\subsubsection{Measurements in work place field}

Measurements simulating a work-place scenario were performed at the truck weighing area of the Feralpi Siderurgica steel plant located in Lonato del Garda (Brescia, Italy). The detector was placed in a reasonable area which could be considered as a candidate area for a ${ }^{241} \mathrm{Am}$ monitor system. A picture of the experiment is shown in Fig. 2. The detector was placed at about $70 \mathrm{~cm}$ from the incoming trucks (roughly the same distance as a standard RPM) 
Fig. 2 Picture of the experimental setup at the Feralpi Siderurgica plant. The neutron detector (within the red rectangle on the right) was placed on a series of stacked pallets to approximately match the center of a typical truck. The detector was placed in proximity to the cargo at the weighing area to simulate a real scenario. As a comparison, on the left of the picture the RPM system employed in the plant is also shown

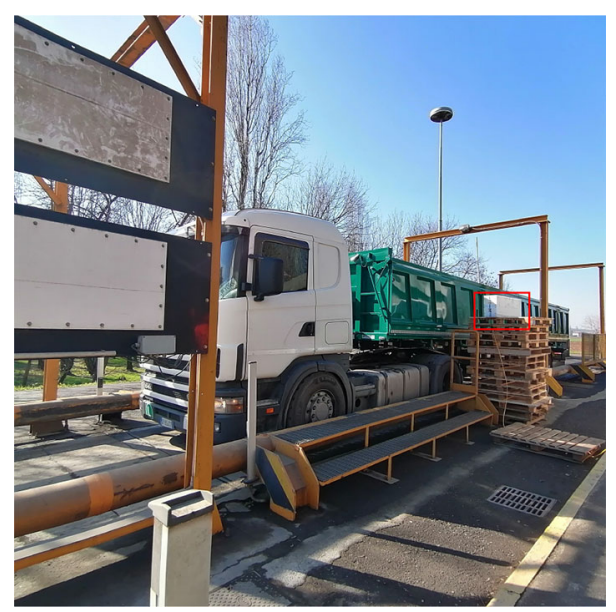

and about $2 \mathrm{~m}$ from the ground, corresponding to nearly half the height of a typical truck. Measurement lasted for few hours and more than 60 incoming loads were monitored. The main goals of the measurements were:

1. Check the possible background attenuation effect when a load is positioned in front of the detector.

2. Estimate a feasible measurement duration that can guarantee reliable results and be compatible with the plant working times.

3. Check the consistence of the outdoor background measurement with the Poisson statistics.

Standard RPM systems for gamma counting experience the background attenuation due to the gamma shielding provided by the cargo material. Conversely, the neutron background is expected to be insensitive to the cargo materials. The first goal was defined to verify this assumption. It should be noted that if strong background attenuation is observed, the hypothesis related to the shielding independence of the technique should be discarded. Thanks to the time discretization of the output file, and by taking note of the arrival and departure time of the incoming trucks, it was possible to verify the cargo shielding effects.

Point (2) is related to the relatively weak neutron emission due to ${ }^{241} \mathrm{Am}$ oxides which prevents the dynamic measurement even with the most efficient neutron detectors on the market. Thus, static measurement is required. The truck weighing procedure at the plant entrance provides in principle with the possibility to perform the neutron measurement without interfering with the standard plant operations. The mean weighing time duration was then estimated.

Point (3) is related to the calculation of the minimum detectable activity. These calculations require some a priori knowledge on the background counts distribution. The outdoor background was then recorded for the off-line statistical analysis.

\section{Results and discussion}

The output information after each measurement session consisted of a series of $8 x 192$ matrices corresponding to the counts collected each ten seconds by the 8 detector sectors. Even 
though there was still the possibility to work with the single sectors alone, for our analysis, the entire detector was considered as a whole, and the eight sector counts were summed up, thus obtaining a series of 192 bins arrays. Each array contained the $10 \mathrm{~s}$ counts for the entire detector length.

This procedure was performed to enhance the counting statistics. However, no information was lost because each sector behaved in the same way as the others within the statistical uncertainties. In particular (1) there was no evidence of a particularly noisy/efficient sector with respect to the others, and (2) neutron thermalization within the HDPE generated an isotropic expanded thermal neutron field passing through the sensitive volume (i.e. both central sectors and lateral sectors were crossed presumingly by the same number of thermal neutrons on average). Moreover, the detector segmentation is rather a peculiar aspect of the device used, and the generality of the discussion does not justify such detector-specific approach.

The 10-s arrays of counts were then summed up for obtaining the total number of counts collected within an arbitrary time integration window, which was chosen considering the quantity of interest. By diving the total number of counts within the integration window by the number of summed arrays times 10, the result becomes expressed in counts per second (CPS).

\subsection{Measurements in laboratory conditions}

The goals of these measurements were (i) evaluate the system sensitivity to a shielded ${ }^{241} \mathrm{Am}$ source, (ii) evaluate the detector response at different distances from the source, and (iii) obtain the signal spectrum for the S/B optimization. The S/B optimization, described in 3.3, led to the calculation of the optimal ROI (bin extremes 62-137), which is already used here as the reference ROI for the points (i) and (ii) calculations.

The obtained arrays after 10-min irradiations are shown in Fig. 3. The shapes of the three spectra are qualitatively superposable, so that for the S/B optimization the shape of the signal contribution can be considered distance-insensitive. The filled area in the histograms corresponds to the chosen ROI. The CPS related to the ROI as a function of the distance are equal to $59 \pm 0.31$ CPS, $22 \pm 0.19$ CPS and $9.3 \pm 0.12$ CPS for $1 \mathrm{~m}, 2 \mathrm{~m}$, and $4 \mathrm{~m}$, respectively. The inverse square law is not fully respected (with respect to the CPS at $2 \mathrm{~m}$, model overestimation of the order of $50 \%$ at $1 \mathrm{~m}$ and model underestimation of the order of $40 \%$ at 4 $\mathrm{m})$. It is assumed that at $1 \mathrm{~m}$ distance the system underestimates the true activity because of the $1.2 \mathrm{~m}$ length of the HDPE moderator, thus the device surface is not completely crossed by the source neutrons (i.e. the field can not be considered expanded). In fact, the detector provider recommends at least a $2 \mathrm{~m}$ distance from the source for a correct measurement. At $4 \mathrm{~m}$ distance, the albedo neutrons might partially enhance the system response. However, the inverse square law can be considered a reasonable and conservative approximation for the further calculations starting from the CPS measured at $2 \mathrm{~m}$ distance $^{2}$.

\subsection{Measurements in work place field}

The 10-s time discretization of the output log file permitted to reconstruct the entire measurement performed at the Feralpi Siderurgica plant with an adequate time precision. With reference to the three points expressed in 2.3.2:

2 The albedo contribution depends on the geometry of the specific facility. In free air, the inverse square law subsists. In other circumstances, the albedo might eventually enhance, not decrease, the detector response. 

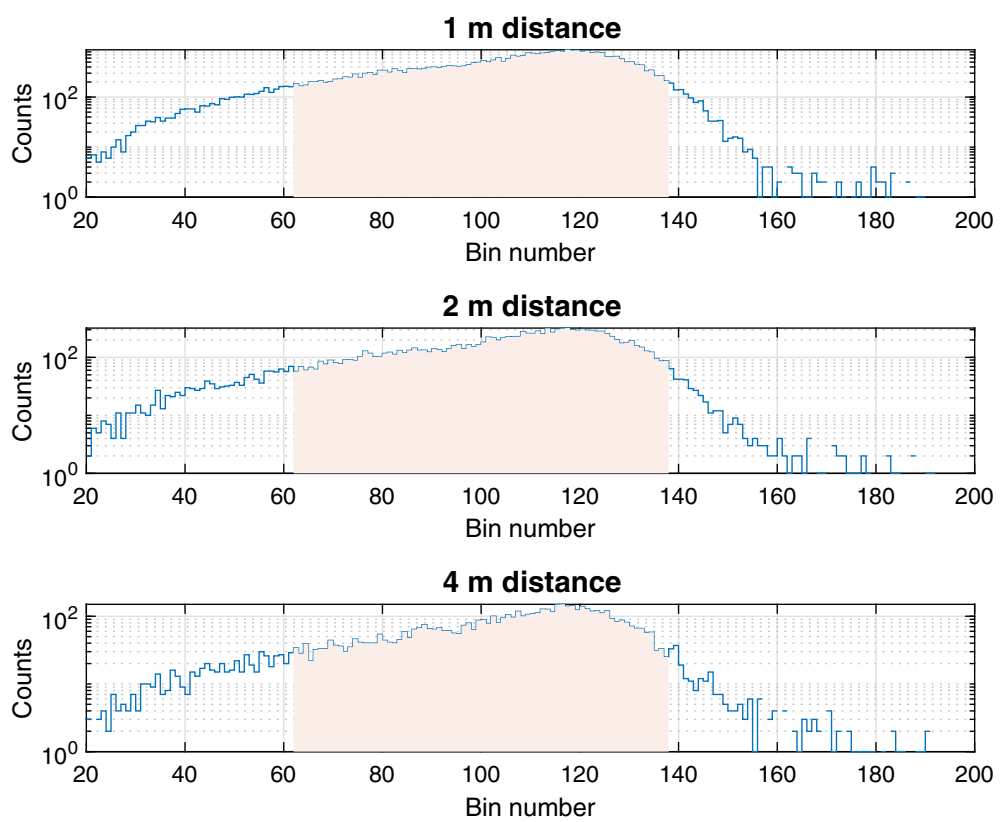

Fig. 3 Comparison of the detector output spectra obtained with the shielded certified ${ }^{241}$ Am source at different distances

1. Shielding effects during the truck weighing were not observed. In particular, the average CPS recorded in the 62-137 ROI during the weighing of the trucks were equal to the pure background CPS (i.e. without the trucks in front of the detector) both around $1.27 \pm 0.01$ CPS.

2. The weighing procedure proved to be a particularly suitable moment for the performance of a static measure, being the only process which involves stationary vehicles inside the steel plant. On the other hand, the time length of the weighting procedure showed to be rather variable. A mean duration of $159 \pm 60 \mathrm{~s}$ was estimated with a 60 trucks population. A time interval of $120 \mathrm{~s}$ showed to be a good estimate of a feasible measurement time and will be taken as a reference time from now on for the calculation of the detector performance.

3. The $\chi^{2}$ test performed on the $120 \mathrm{~s}$ measurement intervals over the entire experiment length returned a $\mathrm{p}$ value equal to 0.54 , which confirmed the consistency of the background counts with the Poisson statistics.

\subsection{Evaluation of the critical detectable activity}

ROI optimization was performed considering the best S/B achievable without excessively losing neutron efficiency. By definition, the $\mathrm{S} / \mathrm{B}$ is equal to the counts recorded with the source in a specific ROI divided by the counts recorded without the source in the same ROI. In this specific case, the detector efficiency is defined as the number of counts recorded at a certain distance from a $37 \mathrm{GBq}^{241} \mathrm{Am}$ point source.

Figure 4 shows the normalized S/B ratio performed channel by channel (since the efficiency depends on the distance, an absolute value of $\mathrm{S} / \mathrm{B}$ is meaningless). The flat region in the middle correspond to a relatively constant $\mathrm{S} / \mathrm{B}$ ratio. ROIs summing up the bins which belong 
Fig. 4 Normalized S/B calculated channel by channel. The best ROI is expected to fall within the almost flat region between 50 and 150 bin numbers

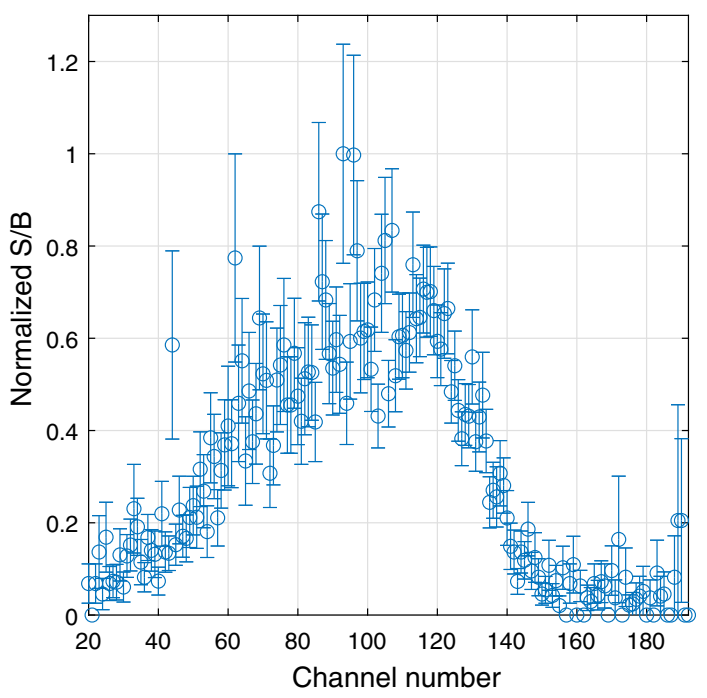

to this region would reasonably show the same S/B. Conversely, the detector sensitivity, in terms of critical detectable activity, strongly depends on the ROI amplitude.

For the evaluation of the critical detectable activity, the Currie theory was followed [8]. The mean background counts obtained in the 120-s time window within the ROI limits, $N_{B}$, is used for evaluating the critical level $L_{c}$ equal to

$$
L_{c}=k_{\alpha} \sigma_{N_{B}}
$$

where $k_{\alpha}$ sets the probability $\alpha$ of the so-called error of the first type, i.e. probability of false positive alarm. The probability $\alpha$ is arbitrary. A $0.5 \%$ probability was assumed adequate for practical applications in steel plants, which translates in a $k_{\alpha}$ coefficient equal to 3.649 for Poisson statistics. $\sigma_{N_{B}}$ is the Poisson standard deviation equal to $\sqrt{N_{B}}$.

By taking into account the neutron emission per unit activity $f$ and the absolute efficiency $\epsilon$, the critical detectable activity for a measurement time $T_{m}$ is then equal to [9]

$$
A_{c}=\frac{L_{c}}{f \epsilon T_{m}}
$$

In the present case, the product $f \epsilon$ was experimentally measured with the ${ }^{241}$ Am source at $2 \mathrm{~m}$ and extrapolated for higher distances basing on the inverse square law.

Figure 5 shows the calculated $A_{c}$ for five different ROIs as a function of the source distance. The best result was obtained for the 62-137 ROI, which will be hereafter used as the reference one.

\subsection{Possible operative configurations for the proposed technique}

The previously described measurements and calculations were aimed at experimentally verifying the technique assumptions and at estimating the $A_{c}$ as a function of the source to detector distance. The last step of the analysis must account for the real case scenario constraints for a RPM system.

It was demonstrated that a 120-s measurement at the weighing area should be a reasonable solution both in terms of geometrical footprint and operational time requirements. The ${ }^{241} \mathrm{Am}$ 
Fig. 5 Values of $A_{c}$ as a function of the distance for 5 different ROIs
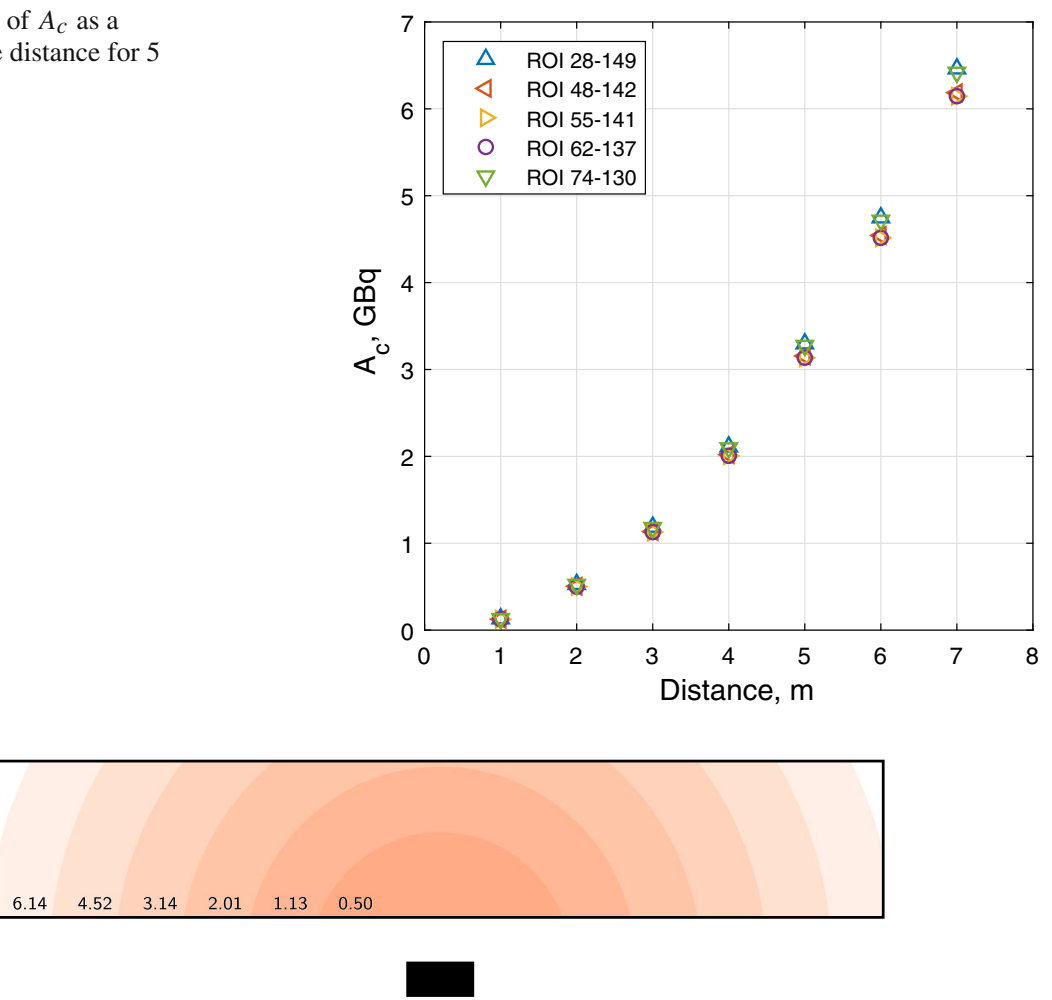

Fig. 6 Schematic cross-sectional view of the truck with the different values of $A_{c}$ highlighted as shaded areas (single-detector setup). The values of $A_{c}$ are expressed in $\mathrm{GBq}$

RPM system could be reasonably installed in this area without interfering with the plant operations. As a reference value, a $70 \mathrm{~cm}$ distance of the detector from the truck was verified to be adequate.

Standard dimensions of the incoming truck can be considered i) height $2.70 \mathrm{~m}$, ii) length $13.60 \mathrm{~m}$, and iii) depth $2.40 \mathrm{~m}$. The first possibility is the installation of a single detector placed beside the load at the center of the cargo length. Figure 6 schematically shows the $A_{c}$ (in $\mathrm{GBq}$ ) in terms of covered cargo area. As it is shown, a $A_{c} \leq 6.14 \mathrm{GBq}$ is guaranteed over a fraction of the total load area higher than $90 \%$.

The single-detector configuration is usually considered particularly inefficient and standard RPM systems always mount at least two detectors. For dynamic measurements, both scintillators are placed along the same longitudinal axis. For the static measurement, the strategy of positioning the detector on different longitudinal axes might be preferred. The dual-detectors configuration is schematically shown in Fig. 7. A $A_{c} \leq 2 \mathrm{GBq}$ is guaranteed over a fraction of the total load area higher than $90 \%$. The obtained $A_{c}$ value could vary by introducing different setup choices (e.g. false positives probability acceptance value, measurement duration, detector type, etc.).

For the calculations, the elastic scattering contribution of the cargo material was neglected. Due to the complex nature of the neutron interactions, the only ways to deal with this shielding-related problem should be i) direct measurement, and/or ii) Monte Carlo simulations. Preliminary Monte Carlo simulations with the MCNPX code [10] were then performed 

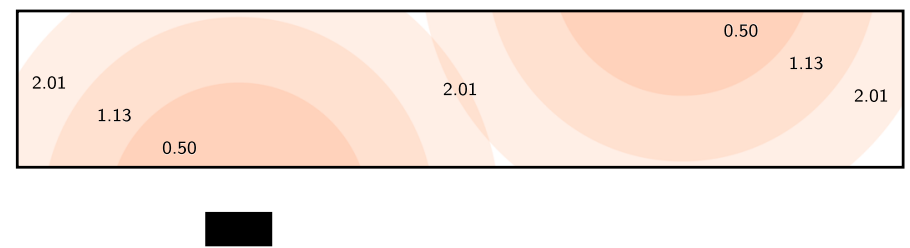

Fig. 7 Schematic cross-sectional view of the truck with the different values of $A_{c}$ highlighted as shaded areas (double-detector setup). The values of $A_{c}$ are expressed in $\mathrm{GBq}$

for the worst case scenarios, i.e. maximum source-to-detector distance, for both single- and dual-detector configurations. The obtained results showed that the system sensitivity was lowered by a factor $40 \%$ in the case of single-detector configuration, and enhanced by a factor $70 \%$ in the case of the dual-detector configuration. In both cases, the result does not change significantly, and it mainly depends on the detector response function $v s$ neutron energy, with only a weak dependence on neutron absorption in scrap. For the generality of the discussion, we thus prefer to neglect this detector-specific perturbation.

\section{Risk evaluation}

In the Tornio accident, the dose received by one worker was estimated to be roughly 10 $\mathrm{mSv}$ by inhalation, and thus the ${ }^{241} \mathrm{Am}$ source melting can be considered a potential hazard. A preliminary risk evaluation was then performed following the example case described in Campi et al. [11]. The same calculations were then repeated considering the critical activity estimated if the proposed neutron detection system is installed at the entrance of the plant.

The hypothesis of the introduction of a ${ }^{241} \mathrm{Am}$ sealed source in the plant must be done considering the presence of a good quality, well maintained RPM system installed at the facility. The International Atomic Energy Agency (IAEA) considers sealed ${ }^{241}$ Am sources as a type $\mathrm{A}_{1}$ material, which allows the transport in type A packages (dose rate at the external surface of the package between $0.005-0.5 \mathrm{mSv} / \mathrm{h}$ ) up to an activity of $10 \mathrm{TBq}$ [12]. For the following calculations, activities of the order of some $\mathrm{Ci}\left(10^{1}-10^{2} \mathrm{GBq}\right)$ are considered.

RPM suppliers usually do not provide data related to the RPM sensitivity to ${ }^{241} \mathrm{Am}$, basically because of the strong shielding dependence of the $59.5 \mathrm{keV}$ gamma rays. We assume that a $5 \mathrm{Ci}(185 \mathrm{GBq})$ source is present in the center of the cargo. Reasonable values of scrap densities range between $0.7-0.9 \mathrm{~g} / \mathrm{cm}^{3}$ [11]. Considering the lower $0.7 \mathrm{~g} / \mathrm{cm}^{3}$ density, the half-value layer of the scrap for ${ }^{241} \mathrm{Am}$ is equal to $0.25 \mathrm{~cm}$, which means that the photon fluence is attenuated by a factor $10^{43}$ at the truck sideboard. Thus, an unshielded $185 \mathrm{GBq}$ ${ }^{241} \mathrm{Am}$ source could pass undetected from the RPM system. If the source in the same position is shielded by a $1 \mathrm{~mm}$ thick lead layer, the attenuation drops to $10^{46}$. It should be noted that the attenuation of a ${ }^{137} \mathrm{Cs}$ source, under the same hypotheses, is of the order of $10^{3}$. The same $10^{3}$ attenuation for a ${ }^{241} \mathrm{Am}$ source is obtained only in the case in which an unshielded source is placed practically in contact to the truck sideboard.

Considering an ${ }^{241} \mathrm{Am}$ source shielded by a $x=1 \mathrm{~mm}$ lead foil entering the radiometric portal, with an activity $A=185 \mathrm{GBq}$, the dose rate $\dot{D}$ in air at $d=0.5 \mathrm{~m}$ is equal to:

$$
\dot{D}=\Gamma \frac{A}{d^{2}} e^{-\mu x} \operatorname{BU}(\mu x)
$$


For the calculations, the mass attenuation coefficients $(\mu)$ were taken from the NIST charts [13], specific gamma exposure constants $(\Gamma)$ from the Oak Ridge National Laboratory tables [14] and build-up factors (BU) from Shimizu et al. [15]. A dose rate of about $5 \mu \mathrm{Sv} / \mathrm{h}$ at 0.5 $\mathrm{m}$ is obtained, which has to be compared to the value of $50 \mu \mathrm{Sv} / \mathrm{h}$ found in [11].

The dose received by the workers before the source melting is due to external irradiation by a point-source, and then it is the same as the dose estimated in [11] scaled by a factor 1/10. Depending on the operation where the accidental exposure occurs, the dose is estimated in between $0.01-0.1 \mu \mathrm{Sv}$. If the alarm at the entrance is not triggered, the most probable fate for the source is to end up in the furnace.

Following the assumptions made in [11] the furnace cabin is provided with an area monitor with a sensitivity of $1 \mu \mathrm{Sv} / \mathrm{h}$, and thus, for a typical $1 \mathrm{~h}$ operation, the dose received by the worker if the alarm is not triggered should be lower than $1 \mu \mathrm{Sv}$. The next quantity to be assessed is the critical ${ }^{241} \mathrm{Am}$ activity concentration in the furnace which does not trigger the alarm. In principle, the $185 \mathrm{GBq}$ source might be detected in this production step, and the dose for the workers was already estimated to be $\leq 0.1 \mu \mathrm{Sv}$ at this stage.

As other actinides, the americium repartition factor is almost $100 \%$ for the slag [16]. The reference nuclide in [11] can thus be considered ${ }^{226} \mathrm{Ra}$. For the accurate evaluation of the critical activity which can trigger the control room area monitor alarm, Monte Carlo calculations have to be performed. However, for a preliminary estimation the results obtained for ${ }^{226} \mathrm{Ra}$ in [11] could be used. The furnace data used are a furnace capacity equal to 100 tons with a slag production per melting equal to 10 tons. Considering the $185 \mathrm{GBq}$ source, the ${ }^{241} \mathrm{Am}$ activity concentration in the slag is equal to about $17430 \mathrm{~Bq} / \mathrm{g}$.

The ${ }^{226} \mathrm{Ra}$ critical concentration, i.e. the minimum concentration which triggers the 1 $\mu \mathrm{Sv} / \mathrm{h}$ alarm, was calculated equal to $1120 \mathrm{~Bq} / \mathrm{g}$. By considering i) the ratio between the fluence-to-dose conversion coefficients of the two nuclides equal to roughly $1 / 9$ $\left(\mathrm{H}^{*}(10) / \Phi_{241_{A m}}=0.51 \mathrm{pSv} \mathrm{cm}{ }^{2}, \mathrm{H}^{*}(10) / \Phi_{226} \mathrm{Ra}=4.38 \mathrm{pSv} \mathrm{\textrm {cm } ^ { 2 }}\right.$ [17]), and ii) the ratio of the attenuation coefficients of the two nuclides equal to roughly 100/1 (for pure iron, $\mu / \rho_{241_{A m}}=1.205 \mathrm{~cm}^{2} / \mathrm{g}, \mu / \rho_{226} \mathrm{Ra}=6.069 \cdot 10^{-2} \mathrm{~cm}^{2} / \mathrm{g}$ [13]), one can reasonably conclude that the $185 \mathrm{GBq}{ }^{241} \mathrm{Am}$ source could pass undetected after the melting step. The Outokumpu cases endorse this hypothesis.

Under this assumption, the last scenario which might involve the exposure of a worker is the slag dump. As a long-lived $\alpha$ emitter, the major risk associated with the dump is the internal contamination through inhalation.

Assuming a typical particulate concentration of $5 \mathrm{mg} / \mathrm{m}^{3}$ [11] and the resulting activity concentration in the slag of $17430 \mathrm{~Bq} / \mathrm{g}$, the local activity concentration would be around 88 $\mathrm{Bq} / \mathrm{m}^{3}$. Looking at derived air concentration limits recently reviewed and published by United States Nuclear Regulatory Commission (USNRC) in the case of occupational inhalation limits for ${ }^{241} \mathrm{Am}$ an air concentration limit of $0.11 \mathrm{~Bq} / \mathrm{m}^{3}$ is provided [18]. Thus locally the ${ }^{241} \mathrm{Am}$ activity concentration would be three orders of magnitude above the recommended limit.

To estimate the dose received by the worker, the model reported by Baldry et al. was used [19]. Calling $D$ the equivalent dose received by the worker:

$$
D=A \cdot C \cdot V \cdot R \cdot e
$$

where $A$ is the activity, $C$ is the dispersion coefficient, $V$ is the reference man breathing rate, $R$ the release fraction and $e$ the dose coefficient of ${ }^{241} \mathrm{Am}$. The dispersion coefficient is evaluated using the Gaussian plume dispersion model [20] following the calculation of Baldry et al. [19]. The dose coefficient for ${ }^{241} \mathrm{Am}$ inhalation is taken from the Italian Act D. Lgs. 230/95 tab. IV-1 [21]. A dose of $1.7 \mathrm{mSv}$ would result in half an hour operation inside the local high concentration area, exceeding the $1 \mathrm{mSv}$ annual dose limit for non 
exposed workers. Even though the generalized approach and the introduced approximations, the obtained result is in line with the accident of Outokumpu (exposure of mSv through inhalation at the slag dump caused by the melting of an ${ }^{241}$ Am activity of the order of some $\mathrm{Ci}$.

The same calculations can be repeated considering the proposed neutron detector system placed at the weighing area of the plant in the dual-detectors configuration. In this case the detection limit at the entrance drops from $185 \mathrm{GBq}$ down to $2 \mathrm{GBq}$. The dose received by the workers is proportionally lowered reaching the value of only $24 \mu \mathrm{Sv}$ at the slag dump. The peculiar aspect of this result is that the $2 \mathrm{GBq}$ value is valid both for unshielded and strongly shielded sources, without any appreciable influence of the equivalent density of the cargo material or of the position of the source with respect to the truck sideboard.

\section{Conclusions}

Recent cases of inadvertently melting of tens-hundreds $\mathrm{GBq}{ }^{241} \mathrm{Am}$ sources at steel plants have caught the attention of the regulatory authorities because of their recurrence and the risk associated to non-exposed workers. Even large activity sources might pass undetected even through the most recent RPM systems and multiple controls. The difficulty in revealing ${ }^{241}$ Am sources mainly comes from its low energy gamma ray emission, which can be easily shielded by the cargo material or by millimeter-thick lead shielding of their packages.

The large majority of ${ }^{241} \mathrm{Am}$ sealed sources is composed by americium oxides encapsulated with other low atomic number materials. $(\alpha, \mathrm{n})$ reactions on these nuclei cause the emission of roughly $10^{3}$ neutrons per second per GBq which can be used to detect ${ }^{241} \mathrm{Am}$ sources at the entrance of a steel plant with a proper neutron sensitive system.

We performed a feasibility study of such system to be installed in a hypothetical steel plant. The ARKTIS M800 detector used is characterized by (1) a rather high sensitivity to $\mathrm{MeV}$ neutrons, and (2) an overall robustness of the design. Thus, the detector can be considered a representative of a class of ${ }^{3} \mathrm{He}$-free devices which could be employed in this type of work-place fields.

Two types of tests were performed. The first set of measurements was performed with a highly shielded certified $37 \mathrm{GBq}{ }^{241} \mathrm{Am}$ source. A second series of measurements was performed directly at the weighing area of a steel plant.

The obtained data permitted to calculate the minimum amount of ${ }^{241} \mathrm{Am}$ activity which can be detected by this system. Considering (1) $120 \mathrm{~s}$ measurement during the weighing, thus without interfering with the standard plant operations, (2) a truck-to-detector distance analogous to that of a standard RPM, (3) an accepted false positive rate of $0.5 \%$, for a single detector configuration $6.14 \mathrm{GBq}$ can be revealed over the $90 \%$ of the load area. In a dual-detectors configuration, under the same conditions, the limit falls to $2 \mathrm{GBq}$. Further improvements in the results are expected. In particular the employment of neutron detectors properly designed for this application with a fine adjustment of the measurement parameters might permit to lower the minimum detectable activity. For the precise dimensioning of the system, in particular the moderator, the elastic scattering effects should be studied in details, since it was shown that these phenomena might both decrease or enhance the specific device response per unit ${ }^{241} \mathrm{Am}$ activity.

A risk evaluation was performed. For a steel plant equipped with standard RPMs and area monitors, an accidental case of a few $\mathrm{Ci}^{241} \mathrm{Am}$ source melting may cause a dose to the worker at the slag dump up to $1-2 \mathrm{mSv}$, in line with the report of a recent accident in 
Finland. For a steel plant equipped with the proposed RPM system, the dose to the worker was estimated to decrease down to $24 \mu \mathrm{Sv}$.

Acknowledgements The authors would like to acknowledge the ARKTIS Radiation Detectors company for providing us with the M800 detector used for the measurements. We also acknowledge the Feralpi Siderurgica company for allowing us to perform the on-site tests at the Lonato del Garda steel plant. The authors want to thank professor Fabrizio Campi for the useful discussions about the risk evaluation study.

Funding Open access funding provided by Politecnico di Milano within the CRUI-CARE Agreement.

Open Access This article is licensed under a Creative Commons Attribution 4.0 International License, which permits use, sharing, adaptation, distribution and reproduction in any medium or format, as long as you give appropriate credit to the original author(s) and the source, provide a link to the Creative Commons licence, and indicate if changes were made. The images or other third party material in this article are included in the article's Creative Commons licence, unless indicated otherwise in a credit line to the material. If material is not included in the article's Creative Commons licence and your intended use is not permitted by statutory regulation or exceeds the permitted use, you will need to obtain permission directly from the copyright holder. To view a copy of this licence, visit http://creativecommons.org/licenses/by/4.0/.

\section{References}

1. IAEA Incident and Trafficking Database (ITDB) . https://www.iaea.org/sites/default/files/20/02/itdbfactsheet-2020.pdf, 2020. Accessed: 2020-06-25 (2020)

2. Online. https://news.cision.com/outokumpu-oyj/r/contaminated-batch-of-recycled-steel-results-inradiation-incident-in-outokumpu-s-tornio-site,c2913902. Accessed: 2020-06-17

3. Online. https://www.reuters.com/article/us-finland-nuclear-outokumpu/outokumpu-steel-plant-infinland-hit-by-four-radiation-incidents-since-july-watchdog-idUSKCN1MQ1RF. Accessed: 2020-0617

4. Sealed Radiation Sources, product information. Document from Nuclitec. http://www.hanshin-eng.co. kr/index/img/5Nuclitec.pdf. Accessed: 2020-06-17

5. W.W. Schulz. The Chemistry of Americium. Technical Information Center Energy Research and Development Administration, (1976)

6. E.F. Shores. Neutron production in several americium compounds (LA-UR-04-3600). (2004)

7. Online. http://www.oecd-nea.org. Accessed: 2020-06-17

8. L.A. Currie, Limits for qualitative detection and quantitative determination. Appl. Radiochem. Anal. Chem. 40, 586-593 (1968)

9. G.F. Knoll. Radiation detection and measurement. Wiley and Sons, Inc., 3rd edition, (1999)

10. X-5 Monte Carlo Team. MCNP - A General Monte Carlo N-Particle Transport Code, Version 5. Volume I: Overview and Theory. Los Alamos National Laboratory, Oak Ridge, April (2003)

11. F. Campi, A.A. Porta, Sensitivity tests and risk evaluation for steelworks portal systems. Radiat. Measurements 39(2), 161-173 (2005)

12. Regulations for the Safe Transport of Radioactive Material. Number SSR-6 (Rev.1) in Specific Safety Requirements. International Atomic Energy Agency, Vienna, (2018)

13. J.H. Hubbell, S.M. Seltzer. X-Ray Mass Attenuation Coefficients: NIST Standard Reference Database 126. https://www.nist.gov/pml/x-ray-mass-attenuation-coefficients. Accessed: 2020-06-18

14. L.M. Unger, D.K. Trubey. Specific gamma-ray dose constants for nuclides important to dosimetry and radiological assessment (ORNL/RSIC-45/Rev1), (1982)

15. Akinao Shimizu, Takashi Onda, Yukio Sakamoto, Calculation of Gamma-ray buildup factors up to depths of $100 \mathrm{mfp}$ by the method of invariant embedding, (III). J. Nucl. Sci. Techno. 41(4), 413-424 (2004)

16. D. Kopsick. Potential recycling of scrap metal from nuclear facilities. Technical support document prepared for the US Environmental Protection Agency, (2001)

17. ICRU Report 57. Journal of the International Commission on Radiation Units and Measurements, os29(2):NP-NP, (2016)

18. Annual limits on intake (ALI) and derived air concentrations (DAC) of radionuclides for occupational exposure

19. K. Baldry, D. Harvey, A. Bishop. Accidental melting of radioactive sources. 01 (2004)

20. Seema Awasthi, Mukesh Khare, Prashant Gargava, General plume dispersion model (GPDM) for point source emission. Environ. Model. Assess. 11(08), 267-276 (2006) 
21. Legislative Decree of Government 17 March 1995 nr. 230 and Subsequent Modifications and Additions (Decreto Legislativo del Governo 17 marzo 1995 nr 230 e Successive Modifiche ed Aggiunte - Attuazione delle direttive 89/618/Euratom, 90/641/Euratom, 92/3/Euratom e 96/29/Euratom in materia di radiazioni ionizzanti), Document written in Italian, (1995) 